PRENATAL DIAGNOSIS

Prenat Diagn 2006; 26: 973-979.

Published online 7 August 2006 in Wiley InterScience

(www.interscience.wiley.com) DOI: 10.1002/pd.1540

\title{
ADAM 12 as a first-trimester maternal serum marker in screening for Down syndrome
}

\author{
Jennie Laigaard ${ }^{1}$, Kevin Spencer ${ }^{2}$, Michael Christiansen ${ }^{1}$, Nicholas J. Cowans ${ }^{2}$, Severin Olesen Larsen ${ }^{1}$, \\ Bent Norgaard Pedersen ${ }^{1}$ and Ulla M. Wewer ${ }^{3}$ \\ ${ }^{1}$ Department of Clinical Biochemistry, Statens Serum Institut, Copenhagen, Denmark \\ ${ }^{2}$ Prenatal Screening Unit, Clinical Biochemistry Department, Harold Wood Hospital, Romford, UK \\ ${ }^{3}$ Institute of Molecular Pathology, University of Copenhagen, Denmark
}

Background A Disintegrin And Metalloprotease 12 (ADAM 12) is a glycoprotein synthesised by placenta and it has been shown to be a potential first-trimester maternal serum marker for Down syndrome (DS) in two small series. Here we analyse further, the potential of ADAM 12 as a marker for DS in a large collection of first-trimester serum samples.

Materials and Methods The concentration of ADAM 12 was determined in $10-14$-week pregnancy sera
from 218 DS pregnancies and 389 gestational age-matched control pregnancies, which had been collected as
part of routine prospective first-trimester screening programs (DS = 105) or as part of previous research studies
(DS = 113). ADAM 12 was measured using a semi-automated time resolved immunofluorometric assay and
median values for normal pregnancies were established by polynomial regression. These medians were then
used to determine population distribution parameters for DS and normal pregnancy groups. Correlation with
previously established PAPP-A and free $\beta$-hCG multiple of the medians (MoMs) and delta nuchal translucency
(NT) were determined and used to model the performance of first-trimester screening with ADAM 12 in
combination with other first-trimester markers at various time periods across the first trimester. The benefits
of a contingent testing model incorporating early measurement of PAPP-A and ADAM 12 were also explored.

Results The maternal serum concentration of ADAM 12 was significantly reduced $(p=0.0049)$ with an overall median MoM of 0.79 in the DS cases and a $\log _{10}$ MoM SD of 0.3734 in the DS cases and 0.3353 in the controls. There was a significant correlation of ADAM $12 \mathrm{MoM}$ in DS cases with gestational age $(r=0.375)$ and the median MoM increased from 0.50 at $10-11$ weeks to 1.38 at 13 weeks. ADAM 12 was correlated with maternal weight $(r$ controls $)=0.283)$, PAPP-A $(r$ (controls $)=0.324, r(\mathrm{DS})=0.251)$ but less so with free $\beta$-hCG $(r$ (controls $)=0.062, r(\mathrm{DS})=0.049)$ and delta NT $(r$ (controls $)=0.110, r(\mathrm{DS})=0.151)$. ADAM 12 was significantly $(p=0.026)$ lower in smokers $(0.87$ vs 1.00$)$ and elevated in Afro-Caribbean women compared to Caucasian women (1.34 vs 1.00).

Population modelling using parameters from this and an earlier study showed that a combination of ADAM 12 and PAPP-A measured at 8-9 weeks and combined with NT and free $\beta$-hCG measured at 12 weeks could achieve a detection rate of $97 \%$ at a $5 \%$ false-positive rate or $89 \%$ at a $1 \%$ false-positive rate. PAPP-A and ADAM 12 alone at 8-9 weeks could identify $91 \%$ of cases at a $5 \%$ false-positive rate. Using this as part of a contingent-screening model to select an intermediate risk group of women for NT and free $\beta$-hCG at 11-12 weeks would enable the detection of $92 \%$ of cases with a $1 \%$ false-positive rate at a cost of providing NT and free $\beta$-hCG for $6 \%$ of women with $94 \%$ of women having completed screening by the 10th week of pregnancy.

Conclusion ADAM 12 in early first trimester is a very efficient marker of DS. In combination with existing markers, it offers enhanced screening efficiency in a two-stage sequential first-trimester screening program or in a contingent-screening model, which may have benefits in health economies where universal access to high quality ultrasound is difficult. More data on early first-trimester cases with DS are required to establish more secure population parameters by which to assess further the validity of these models. Copyright (C) 2006 John Wiley \& Sons, Ltd.

KEY WORDS: prenatal screening; PAPP-A; free $\beta$-hCG; growth factors; ADAM 12; aneuploidy; trisomy 21

\section{INTRODUCTION}

ADAM 12 is the secreted form of A Disintegrin And Metalloprotease 12, a glycoprotein synthesised by the placenta and secreted throughout pregnancy (Gilpin et al., 1998; Shi et al., 2000). ADAM12 has

*Correspondence to: Kevin Spencer, Prenatal Screening Unit, Clinical Biochemistry Department, Harold Wood Hospital, Gubbins Lane, Romford, UK. E-mail: KevinSpencer1@ aol.com a proteolytic function against insulin-like growth factor binding protein, IGFBP-3 and IGFBP-5 (Loechel et al., 2000) and regulates the bioavailability and action of IGF-II and IGF-I (Rosenfeld and Roberts, 1999; Lamson et al., 1993).

In small series of maternal serum samples, ADAM 12 has been shown to be reduced in the first trimester in Down syndrome (DS) (Laigaard et al., 2003, 2006) and trisomy 18 pregnancies (Laigaard et al., 2005b) as well as in women later developing preeclampsia (Laigaard 
et al., 2005a). The discriminatory efficiency of ADAM 12 appears to decrease with advancing gestation and it has been suggested, based on a very small number of samples, that ADAM 12 is not useful in gestational week 11-13 (Laigaard et al., 2006), at a time when serum screening is often performed in conjunction with nuchal translucency (NT) as part of the combined screening test (Spencer et al., 2003a).

In order to develop further our understanding of ADAM 12 and its potential value as a first-trimester marker of DS, we undertook a large-scale study of cases and controls collected as part of a first-trimester combined ultrasound and biochemical screening program for chromosomal anomalies (OSCAR) (Spencer et al., 2003a).

\section{MATERIALS AND METHODS}

All women booked for maternity care at Harold Wood Hospital, Essex (between June 1998 and December 2003), King George Hospital, Goodmayes (between July 2001 and December 2003), Kent and Canterbury Hospital, Canterbury (between July 2002 and December 2003), William Harvey Hospital, Ashford (between July 2002 and December 2003) and Queen Elizabeth The Queen Mother's Hospital, Margate (between July 2002 and December 2003) were offered screening for DS by a combination of fetal NT and maternal serum free $\beta$-hCG and pregnancy associated plasma protein-A (PAPP-A) at $11^{+0}$ to $13^{+6}$ weeks. The maternal serum free $\beta$ hCG and PAPP-A were measured using the Kryptor analyser (Brahms AG, Berlin) and the performance of this assay has been described previously (Spencer et al., 1999). An ultrasound examination was carried out to measure the fetal NT and crown-rump length (CRL) and to diagnose any major structural defects. All scans were carried out by sonographers who had obtained the Fetal Medicine Foundation certificate of competence in the 11-14 weeks scan (www.fetalmedicine.com). Patient specific risks were calculated by a multivariate approach using population parameters established in large-scale retrospective studies and prospective studies (Snijders et al., 1998; Spencer et al., 1999) and the maternal age and gestational related risk of DS at the time of screening (Snijders et al., 1999). Women with a risk of $>1: 300$ were offered invasive testing to determine the fetal karyotype. Data on pregnancy outcome were obtained from the cytogenetics laboratories, the National Down's Syndrome Cytogenetic Register, and the maternity units in which the fetus was delivered. From amongst this population of 45257 singleton pregnancies screened some 105 cases with DS were ascertained. Of the DS cases, 96 were identified by the combined screening program and nine were live-born cases missed by the program. As a control group, 389 samples from across the gestational ranges 10-14 weeks were selected. All samples had been analysed for routine biochemical markers within $1 \mathrm{~h}$ of sample collection and then stored at $4{ }^{\circ} \mathrm{C}$ prior to archive storage at $-20{ }^{\circ} \mathrm{C}$ or $-40{ }^{\circ} \mathrm{C}$ at the end of the day. The cases with DS were further supplemented with a series of 113 samples collected as part of previous research studies (Spencer et al., 2002). Table 1 shows the distribution of cases and controls by gestational week. In all cases, gestational age at sample collection (median controls $=85$ days; median cases $=$ 88 days) was determined by CRL measurement (median controls $=56.9 \mathrm{~mm}$; median cases $=62.0 \mathrm{~mm}$ ). Samples had on average been frozen and thawed as aliquots twice (range 1-3). The control group had a median maternal age of 29.5 years while that for the group of cases was 37.0 years. The maternal weight of the two groups was similar (median controls $=65.0 \mathrm{~kg}$; median cases was $63.5 \mathrm{~kg}$ ). The proportion of smokers in the controls was $17 \%$ in the controls and $10 \%$ amongst the cases. The group was predominantly of Caucasian origin ( $85 \%$ in the controls and $83 \%$ in the cases). All samples were collected as part of projects approved by relevant local ethics committees or were obtained as part of routine screening services for which patients gave written consent for excess diagnostic material to be used for research purposes.

In order to better assess how ADAM 12 changed in normal pregnancy into the second trimester, a series of 362 second trimester samples collected between the 14th and 19th week of gestation were also assessed (Christiansen et al., 2006).

ADAM 12 was measured by a semi-automated time resolved immunofluorometric assay using the AutoDelfia platform (PerkinElmer, Turku, Finland) as previously described (Laigaard et al., 2005a). Briefly

Table 1 -Distribution of cases and controls by gestational age and study

\begin{tabular}{|c|c|c|c|c|c|}
\hline \multirow[b]{2}{*}{$\begin{array}{l}\text { Gestational } \\
\text { week }\end{array}$} & \multicolumn{3}{|c|}{ Down } & \multicolumn{2}{|c|}{ Controls } \\
\hline & $\begin{array}{l}\text { Laigaard } \\
\text { et al., } \\
2003\end{array}$ & $\begin{array}{l}\text { This study } \\
\text { research } \\
\text { series }\end{array}$ & $\begin{array}{c}\text { This study } \\
\text { routine } \\
\text { series }\end{array}$ & $\begin{array}{l}\text { Laigaard } \\
\text { et al., } \\
2003\end{array}$ & $\begin{array}{l}\text { This } \\
\text { study }\end{array}$ \\
\hline 7 & 3 & 0 & 0 & 1 & 0 \\
\hline 8 & 3 & 0 & 0 & 26 & 0 \\
\hline 9 & 7 & 0 & 0 & 52 & 0 \\
\hline 10 & 2 & 0 & 3 & 36 & 52 \\
\hline 11 & 3 & 23 & 17 & 21 & 117 \\
\hline 12 & 0 & 60 & 49 & 12 & 116 \\
\hline 13 & 0 & 30 & 36 & 6 & 104 \\
\hline
\end{tabular}


recombinant human ADAM 12 was used for standardisation. A previously described (Gilpin et al., 1998) monoclonal capture antibody (6E6) was coated onto the surface of microtitre plates after pre-washing the coated plates; $50 \mathrm{uL}$ of sample and $50 \mathrm{uL}$ of AutoDelfia multibuffer were added to each well followed by a 2-h room temperature incubation and four cycles of wash with multibuffer. The detection antibody was a previously described (Gilpin et al., 1998) monoclonal (8F8) which was biotinylated (Laigaard et al., 2005a). The biotinylated detection antibody was added to each well and incubated for $1 \mathrm{~h}$ at room temperature followed by a further four cycles of wash. Europium labelled streptavidin was added to each well and incubated for $1 \mathrm{~h}$ at room temperature followed by three wash cycles and the addition of enhancement solution and counting of immunofluoresence after $10 \mathrm{~min}$. The analytical performance of this assay has been previously described (Laigaard et al., 2005a).

Statistical analysis of data was performed using Microsoft Excel 2000 and Analyse-It (Smart Software, Leeds, UK), a statistical software add-in. To correct for biochemical marker variations with gestational age, each value was converted to a multiple of the median (MoM) for unaffected pregnancies at the same gestational age using either previously established relationships for PAPP-A and free $\beta$-hCG (Ong et al., 2000) or as established for ADAM 12 in this study. Regression analysis was carried out to derive the relationship between ADAM 12 and gestational age. Correction of each MoM for maternal weight was performed as previously described (Spencer et al., 2003b) and as described for ADAM 12. Goodness of fit to a Gaussian distribution of $\log _{10}$ ADAM $12 \mathrm{MoM}$ in the control group and the Down group was confirmed using normal probability plots and the Shapiro-Wilk test. NT was normalised for gestational age by conversion to delta NT as previously described (Spencer et al., 2003c). Marker correlations were determined using Pearson's correlation coefficient.

The performance of various marker combinations as potential screening procedures was examined using standard statistical modelling techniques (Royston and Thompson, 1992). Using the observed population parameters for ADAM 12 and those for PAPP-A and free $\beta$-hCG from Spencer et al. (2003d) and for delta NT from Spencer et al. (2003c), a series of random MoM or delta NT values were selected from the distributions in unaffected and affected pregnancies. These values were then used to calculate likelihood ratios for the combinations. The likelihood ratios were then used together with the age related risk of DS at 12 weeks of gestation (Snijders et al., 1999) to calculate the expected detection rate of affected pregnancies at various false-positive rates, in a population with the maternal age distribution of pregnancies in England and Wales for an age standardised population with a mean age of 27.0 (SD 5.5) using the methodology and parameters described in Cuckle et al., 2004.

To enhance the modelling of gestational age-dependant detection rates, we incorporated ADAM 12 data from Down cases and controls collected between 8 and 11 weeks from a previous study (Laigaard et al., 2003) to allow an analysis of the temporal relationship of ADAM 12 across the first trimester. Table 1 summarises the origin and gestation week distributions of the cases and controls from the various sample groups. We utilised the temporal relationship for free $\beta-\mathrm{hCG}$ and PAPP-A as described in previous studies (Spencer et al., 2002, 2003d). Using the measured parameters for ADAM 12, the correlations established in this study and the published parameters for free $\beta$-hCG and PAPP-A (Spencer et al., 2002, 2003d) we modelled the expected detection rate at various gestations across the first trimester using various marker combinations incorporating ADAM 12.

The performance of contingent, stepwise screening using the combination of PAPP-A and ADAM 12 in week 8-9 to select the pregnancies that may benefit from NT screening in combination with free $\beta$ hCG in week 11-13, was assessed as previously described for PAPP-A and $\beta$ hCG (Christiansen and Larsen, 2002). The precise calculations were performed as a Monte Carlo simulation where initially 100000 a priori risk values were established for cases with DS and for normal cases using age dependent risk values (Cuckle et al., 1987) and a standard age distribution for birth giving mothers (van der Veen et al., 1997). These risk values were then combined with the same number of simulated normally distributed marker values for DS and normal cases allowing correlation between all markers involved. Risk values were subsequently calculated combining likelihood ratio values with the a priori risk values, and it was determined in step 1 for each case whether a positive result (risk $>1: 65$ ) leading to immediate amniocentesis/CVS or a negative result (risk $<1: 1000$ ) leading to no further action was reported. For a number of cases, intermediate results $(1: 1000<$ risk $<1: 65)$ were calculated leading to further screening in step 2 taking account also of the marker values found for step 1. In the final step, a risk of $1: 400$ for giving birth to a DS child was considered the cutoff for invasive diagnostic procedures.

\section{RESULTS}

In normal pregnancies, we found that ADAM 12 concentrations declined with gestation through the first trimester to reach a trough in the early second trimester from which ADAM 12 then began to increase. The best fit to the data was described by a third order polynomial of the form Median ADAM $12 \mathrm{ug} / \mathrm{L}=$ $0.0009 \times$ GA $3-0.2206 \times$ GA $2+14.271 \times$ GA -82.755 with GA being gestational age in days weighted by the number of cases in each gestational week (Figure 1).

Using the median curve derived above, the overall control population showed a median ADAM 12 MoM of 0.99 . When the distribution of $\log _{10}$ MoM ADAM 12 was examined in the first-trimester control case, the mean $\log _{10}$ ADAM 12 was -0.002 with a standard deviation of 0.3485 . In the Down cases the overall median ADAM 12 MoM was 0.79, with a median of 0.65 in the cases from routine screening and 0.86 in the research study series. When the distribution of $\log _{10}$ 


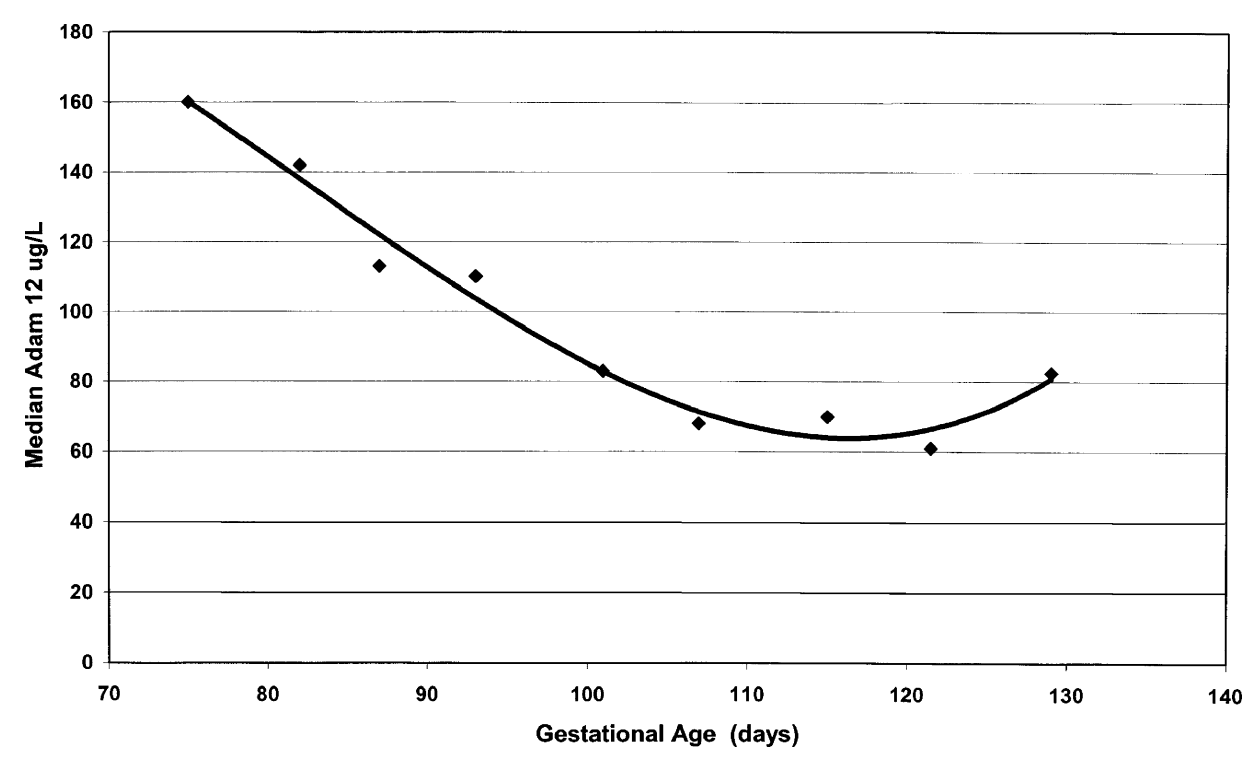

Figure 1 - Variation of ADAM 12 with gestational age
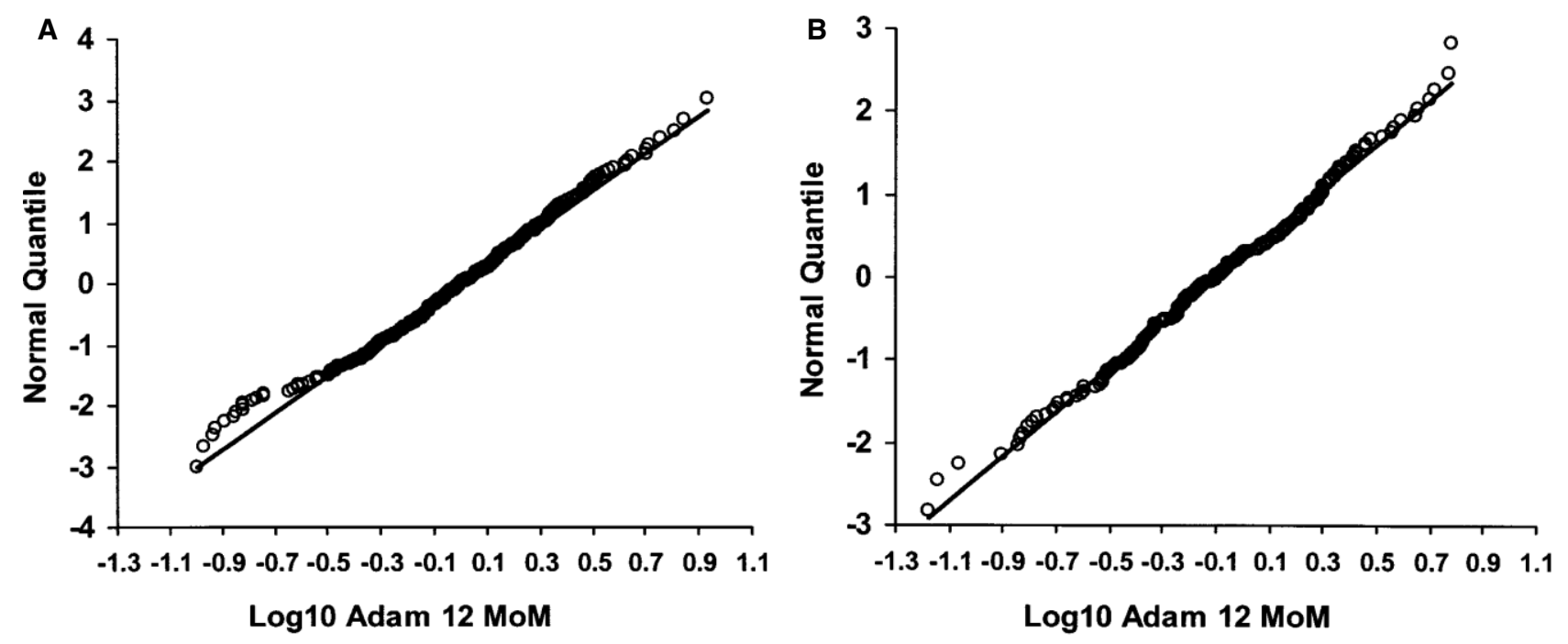

Figure 2-(a) Normal probability plot for $\log _{10}$ ADAM $12 \mathrm{MoM}$ in unaffected pregnancies. (b) Normal probability plot for Log 10 ADAM 12 MoM in Down pregnancies

MoM ADAM 12 was examined in the Down cases the mean $\log _{10}$ ADAM 12 was -0.095 with a standard deviation of 0.3694 . ADAM 12 MoM fitted reasonably well to a Gaussian distribution after $\log _{10}$ transformation (Figure 2). When the $\log _{10}$ ADAM $12 \mathrm{MoM}$ in the control group and the Down group were compared using $t$-tests of unequal variance the mean in the Down group was significantly lower $(p=0.0049)$ than in the control group.

In the control population, there was a significant negative correlation of ADAM 12 MoM with maternal weight $(r=0.283)$ and a suitable reciprocal weight correction formula was derived to correct for this (Corrected $\mathrm{MoM}=$ Measured $\mathrm{MoM} /\left(\left(50.00^{*} 1 /\right.\right.$ weight $)$ $+0.2272)$. When corrected for maternal weight the overall median for the cases was still 0.79 with the median for the routine screening series being 0.67 and research study series 0.87 . This difference was not statistically significant $(p=0.059)$. Weight corrected SD's were 0.3353 in the controls and 0.3734 for T21 cases. Correlation analysis was performed after exclusion of outliers outside of $\pm 3 \mathrm{SD}$. Correlation of weight corrected $\log _{10}$ ADAM 12 MoM with $\log _{10}$ PAPP-A MoM in the control series was 0.324 and 0.251 in the Down cases. Correlation of weight corrected $\log _{10}$ ADAM 12 MoM with $\log _{10}$ free $\beta$-hCG MoM in the control series was 0.062 and 0.049 in the Down cases. Correlation of weight corrected $\log _{10}$ ADAM 12 MoM with delta NT in the control series was 0.110 and 0.151 in the Down series.

In the control group, the median MoM ADAM 12 was significantly lower in women who smoked compared with non-smokers (0.87 vs $1.00 ; p=0.026)$. In the control group, there was an apparent higher median 


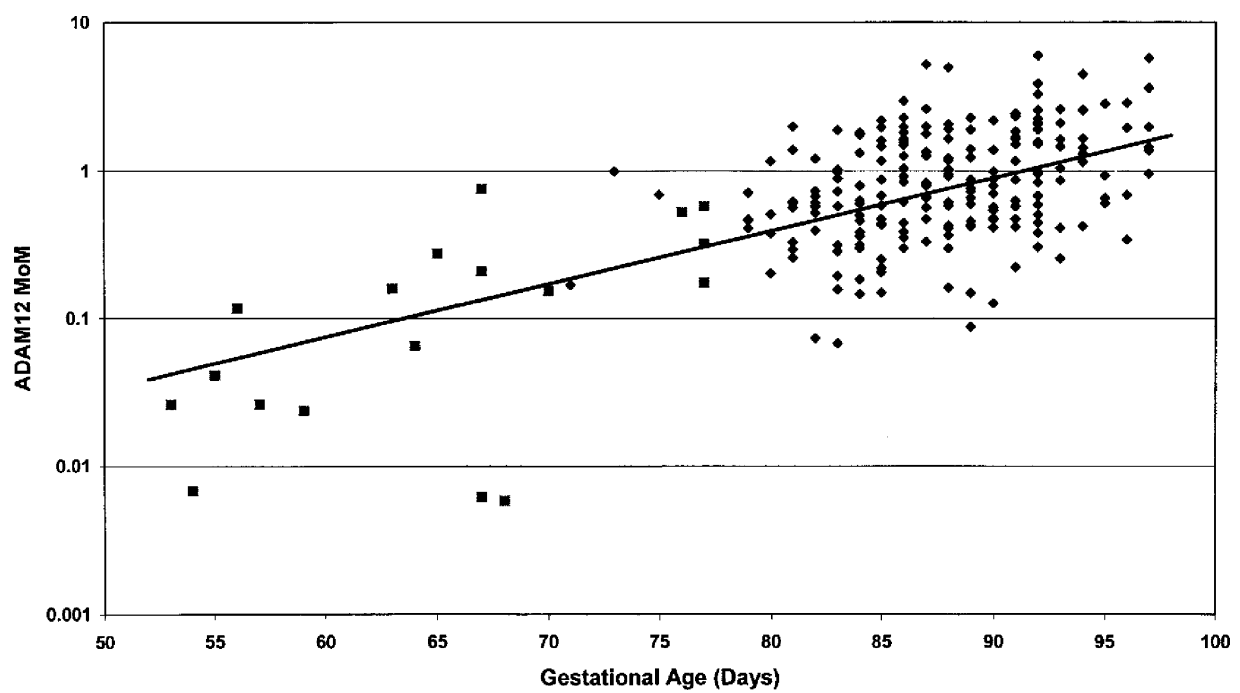

Figure 3-ADAM $12 \mathrm{MoM}$ in cases with Down syndrome in the first trimester from the new study $(\diamond)$ and from the original Laigaard et al., 2003 study (ם). The solid line is the regression line

weight corrected MoM of 1.34 for ADAM 12 in the group of Afro-Caribbean women $(n=19)$ compared with 0.96 in the group of Asian women $(n=38)$ and 1.00 in the Caucasian group $(n=318)$.

When the median ADAM 12 MoM was examined in the DS group with gestational age a significant association was found (Figure 3). The correlation coefficient between $\log _{10}$ ADAM 12 MoM and gestational days was 0.6329 . When analysed by completed gestational week the median MoM was 0.59 at 10 weeks $(n=3), 0.49$ at 11 weeks $(n=39), 0.74$ at 12 weeks $(n=108)$ and 1.38 at 13 weeks $(n=64)$. Overall, taking the group within the 10th and 11th completed week, the median ADAM 12 MoM in cases was 0.50 with a $\log _{10}$ SD after maternal weight correction of 0.325 in cases and 0.290 in controls. In the 12th and 13th completed week, the median ADAM 12 MoM in cases was 0.93 with a $\log _{10}$ $\mathrm{SD}$ of 0.360 in cases and 0.361 in controls. From the previously published series (Laigaard et al., 2003), the median in the 15 cases at $7-10$ weeks of gestation was 0.06 with a $\log _{10}$ SD of 0.6256 in cases and 0.2929 in the 115 controls. The median regression line was $\log _{10} \mathrm{MoM}=0.03599 \times$ Gestational Day -3.27112 .

The measured gestational age-specific parameters for ADAM 12 as described above and those for the other biochemical markers from (Spencer et al., 2002, 2003d) used in the simulation exercise are summarised in Table 1. Table 3 shows the results of modelling detection rates at various false-positive rates when the biochemical markers are combined with maternal age in different gestational weeks and when also combined with NT measurement at 12 weeks of gestation.

The performance of contingent screening is outlined in Figure 4. In accordance with the performance data in Table 2, the large majority, $81.5 \%$ of DS cases, would have a risk $>1: 65$ and consequently be offered

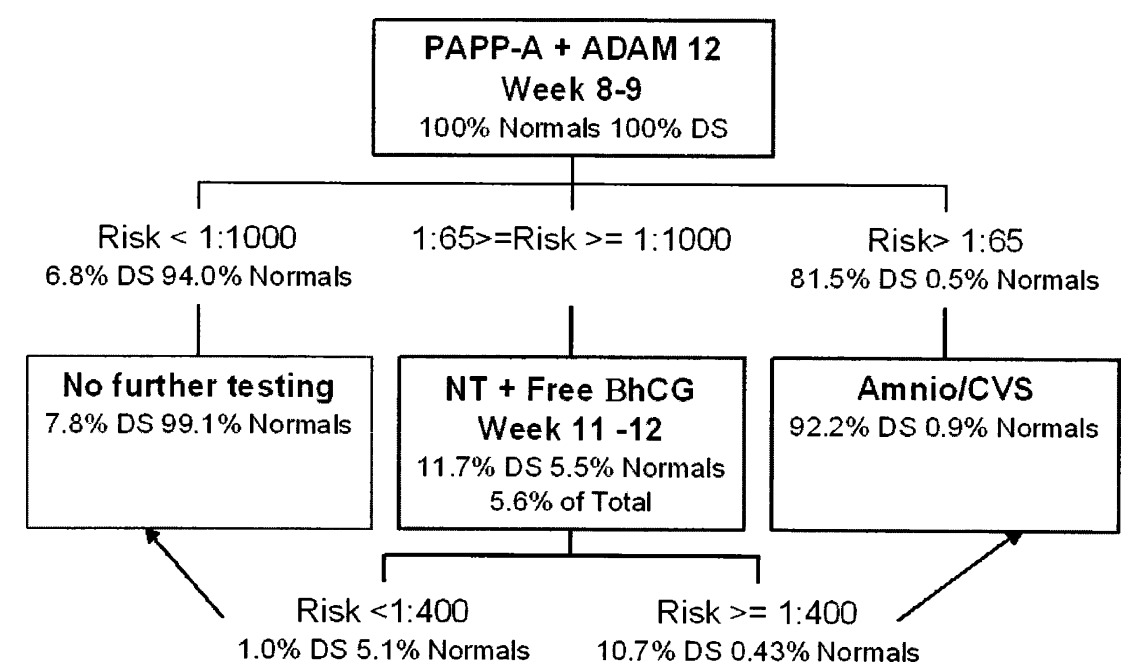

Figure 4-Contingent screening using PAPP-A and ADAM 12 measured at $8-9$ weeks, with the selection of women with risks between $1: 65$ and $1: 1000$ for further testing at $11-12$ weeks with NT and free $\beta$-hCG 
Table 2-Summary of population parameters used in the simulation study

\begin{tabular}{lccc}
\hline Period & $\begin{array}{c}\text { Mean } \log _{10} \\
\text { MoM T21 }\end{array}$ & $\begin{array}{c}\text { SD } \\
\text { T21 }\end{array}$ & $\begin{array}{c}\text { SD } \\
\text { control }\end{array}$ \\
\hline ADAM 12 8-9 weeks & -1.222 & 0.6256 & 0.2929 \\
ADAM 12 10-11 weeks & -0.3010 & 0.3251 & 0.2900 \\
ADAM 12 12-13 weeks & -0.0320 & 0.3603 & 0.3612 \\
Free $\beta$-hCG 8-9 weeks & 0.1535 & 0.2858 & 0.2618 \\
Free $\beta$-hCG 10-11 weeks & 0.2683 & 0.2858 & 0.2618 \\
Free $\beta$-hCG 12-13 weeks & 0.3857 & 0.2858 & 0.2618 \\
PAPP-A 8-9 weeks & -0.4529 & 0.3027 & 0.2362 \\
PAPP-A 10-11 weeks & -0.3369 & 0.3027 & 0.2362 \\
PAPP-A 12-13 weeks & -0.2445 & 0.3027 & 0.2362 \\
NT 12-13 weeks & 0.3050 & 0.2350 & 0.1200 \\
\hline
\end{tabular}

Correlation $(r)$ values

\begin{tabular}{lll}
\hline Marker & T21 & Controls \\
\hline PAPP-A vs ADAM 12 & 0.2512 & 0.3240 \\
Free $\beta$-hCG vs ADAM 12 & 0.0490 & 0.0624 \\
PAPP-A vs Free $\beta$-hCG & 0.1250 & 0.2178 \\
PAPP-A vs NT & 0 & 0 \\
Free $\beta$-hCG $v s$ NT & 0 & 0 \\
ADAM 12 $v s$ NT & 0.1510 & 0.1100 \\
\hline
\end{tabular}

CVS without further-and later-screening, whereas 94\% of the non-affected pregnancies would have a risk $<1: 1000$ and consequently not be offered further screening as such screening would improve DR marginally. Only $5.6 \%$ of all pregnancies would be offered NT and free $\beta$ hCG screening. The overall detection for DS would be $92 \%$ for a mere $0.9 \%$ false-positive rate. And more than $90 \%$ of all pregnant women would receive a final risk estimate before week 10 .

\section{DISCUSSION}

The present study is the first large-scale study of the performance of ADAM 12 as a first-trimester marker for DS and the results confirm the findings in the previously published small series (Laigaard et al., 2003, 2006). It is also confirmed that the difference between ADAM 12 levels in DS and control pregnancies decrease going from week 10-11 to week 12-13, but, surprisingly, even though the difference is negligible in week 12-13, the marker is still useful in combination with PAPP-A and free $\beta$ hCG (Table 3 ). This is most likely a result of the significant correlation with PAPP-A (Table 2).

The previously expressed doubt about the suitability of ADAM 12 as a marker in the OSCAR setting (Laigaard et al., 2006) does not seem to be well founded. However, ADAM 12 has the best discriminatory efficiency early in the first trimester (Table 2). In that respect, the marker behaves in a manner similar to PAPP-A (Spencer et al., 2002, 2003d; Christiansen and Jaliashvili, 2003), ProMBP (Christiansen et al., 1999, 2004) and SP1 (Qin et al., 1997).

From the performance of ADAM 12 in combination with PAPP-A as the first step-performed in week $8-9$-in a contingent screening model, it is seen that a very impressive overall screening performance can be obtained with less than $6 \%$ being offered NT screening and more than $90 \%$ of women having a final risk result before week 10 . However, from a health economic point of view the benefit to detection of adding the second biochemistry test at 12 weeks (free $\beta$ hCG) may be questionable.

Thus, one potential role for ADAM 12 may be in health economies where universal access to high quality ultrasound and NT measurement is difficult either due to lack of availability of trained sonographers or when cost is a major consideration. In this regard, a simple blood test at 8-9 weeks could be used in the Contingent type model along with PAPP-A. One perceived disadvantage to such a system may be that women in some health economies do not present this early for screening; however, in Europe there is a growing desire to introduce early biochemical screening which takes into account the additional detection brought about by early screening with PAPP-A, and which enables a combined risk and counselling to be provided at the time of the NT examination.

The molecular background for the reduced maternal serum level of ADAM 12 in DS pregnancies has not been established, so the clinical use of the marker should

Table 3-Simulated population detection rates (\%) at various screen-positive rates using biochemistry at 8-9 weeks, 10-11 weeks or $12-13$ weeks, and NT (when included) at 12 weeks

\begin{tabular}{|c|c|c|c|c|c|c|c|c|c|}
\hline \multirow{2}{*}{$\frac{\text { Screen-positive rate }}{\text { Combination (weeks) }}$} & \multicolumn{3}{|c|}{$5 \%$} & \multicolumn{3}{|c|}{$3 \%$} & \multicolumn{3}{|c|}{$1 \%$} \\
\hline & $8-9$ & $10-11$ & $12-13$ & $8-9$ & $10-11$ & $12-13$ & $8-9$ & $10-11$ & $12-13$ \\
\hline ADAM 12 \& maternal age & 90 & 48 & 43 & 87 & 42 & 32 & 63 & 24 & 19 \\
\hline Free $\beta$-hCG \& maternal age & 30 & 39 & 52 & 23 & 31 & 43 & 14 & 19 & 27 \\
\hline PAPP-A \& maternal age & 56 & 43 & 35 & 48 & 36 & 28 & 30 & 20 & 17 \\
\hline PAPP-A, Free $\beta$-hCG $\&$ age & 64 & 61 & 64 & 55 & 52 & 56 & 39 & 37 & 40 \\
\hline PAPP-A, ADAM 12 \& age & 91 & 58 & 50 & 89 & 49 & 41 & 69 & 32 & 27 \\
\hline ADAM 12 , Free $\beta$-hCG \& age & 90 & 57 & 62 & 87 & 49 & 53 & 66 & 34 & 38 \\
\hline Free $\beta$-hCG, ADAM 12, PAPP-A \& age & 92 & 70 & 74 & 88 & 63 & 66 & 71 & 47 & 51 \\
\hline Free $\beta$-hCG, PAPP-A, NT \& age & 86 & 85 & 86 & 82 & 80 & 82 & 72 & 71 & 73 \\
\hline PAPP-A, ADAM 12, NT \& age & 97 & 84 & 80 & 95 & 80 & 76 & 87 & 70 & 66 \\
\hline Free $\beta$-hCG, ADAM 12 , NT \& age & 96 & 84 & 85 & 94 & 80 & 81 & 87 & 71 & 72 \\
\hline Free $\beta$-hCG, PAPP-A, ADAM 12, NT \& age & 97 & 89 & 90 & 95 & 86 & 86 & 89 & 78 & 78 \\
\hline
\end{tabular}


be performed with care and it is imperative that the marker is only used in a setting with full follow-up and continuous monitoring of screening parameters.

Further studies should be directed at documenting the retrospective and prospective efficiency of the markers, particularly in conjunction with other markers in the early first trimester.

\section{ACKNOWLEDGEMENT}

This study was supported in part by a grant from NHS R\&D (RF4: Risk Assessment in Pregnancy) to Professor Kevin Spencer.

\section{REFERENCES}

Christiansen M, Jaliashvili I. 2003. Total pregnancy-associated plasma protein A-a first trimester maternal serum marker for Down's syndrome: clinical and technical assessment of a polymonoclonal enzyme immunoassay. Scand J Clin Lab Invest 63: 407-415.

Christiansen M, Larsen SO. 2002. An increase in cost-effectiveness of first trimester maternal screening programmes for fetal chromosome anomalies is obtained by contingent testing. Prenat Diagn 22: 482-486.

Christiansen M, Oxvig C, Wagner JM, et al. 1999. The proform of eosinophil major basic protein: a new maternal serum marker for Down syndrome. Prenat Diagn 19: 905-910.

Christiansen M, Larsen SO, Oxvig C, et al. 2004. Screening for Down's syndrome in early and late first and second trimester using six maternal serum markers. Clin Genet 65: 11-16.

Christiansen M, Spencer K, Laigaard J, Cowans NJ, Larsen SO, Wewer UM. 2006. ADAM 12 as a second trimster maternal serum markers in screening for Down's syndrome. Prenat Diagn 26: (in press).

Cuckle HS, Wald NJ, Thompson SG. 1987. Estimating a woman's risk of having a pregnancy associated with Down's syndrome using her age and serum alpha-fetoprotein level. Br J Obstet Gynaecol 94: 387-402.

Cuckle H, Aitken D, Goodburn S, Senior B, Spencer K, Standing S. 2004. Age-standardisation for monitoring performance in Down's syndrome screening programmes. Prenat Diagn 24: 851-856.

Gilpin BJ, Loechel F, Mattei MG, Engvall E, Albrechtsen R, Wewer UM. 1998. A novel, secreted form of human ADAM 12 (meltrin alpha) provokes myogenesis in vivo. J Biol Chem 273: 157-166.

Laigaard J, Sorensen T, Frohlich C, et al. 2003. ADAM12: a novel first-trimester maternal serum marker for Down syndrome. Prenat Diagn 23: 1086-1091.

Laigaard J, Sorensen T, Placing S, et al. 2005a. Reduction of the disintegrin and metalloprotease ADAM12 in preeclampsia. Obstet Gynecol 106: 144-149.

Laigaard J, Christiansen M, Frohlich C, Pedersen BN, Ottesen B, Wewer UM. 2005b. The level of ADAM12 in maternal serum is an early first-trimester marker of fetal trisomy 18. Prenat Diagn 25: $45-46$.
Laigaard J, Cuckle H, Wewer UM, Christiansen M. 2006. Maternal serum ADAM12 levels in Down's and Edwards' syndrome pregnancies at 9-12 weeks gestation. Prenat Diagn 26: In Press.

Lamson G, Giudice LC, Cohen P, et al. 1993. Proteolysis of IGFBP3 may be a common regulatory mechanism of IGF action in vivo. Growth Regul 3: 91-95.

Loechel F, Fox JW, Murphy G, Albrechtsen R, Wewer UM. 2000. ADAM 12 cleaves IGFBP-3 and IGFBP-5 and is inhibited by TIMP-3. Biochem Biophys Res Commun 278: 511-515.

Ong CYT, Liao AW, Spencer K, Munim S, Nicolaides KH. 2000. First trimester maternal serum free $\beta$ human chorionic gonadotrophin and pregnancy associated plasma protein $\mathrm{A}$ as predictors of pregnancy complications. BJOG 107: 1265-1270.

Qin QP, Christiansen M, Nguyen TH, Sorensen S, Larsen SO, Norgaard-Pedersen B. 1997. Schwangerschaftsprotein 1 (SP1) as a maternal serum marker for Down syndrome in the first and second trimesters. Prenat Diagn 17: 101-108.

Rosenfeld RG, Roberts CT (eds). 1999. The IGF System. Molecular Biology, Physiology, and Clinical Applications. Humana Press: Totowa, NJ; 1-787.

Royston P, Thompson SG. 1992. Model based screening by risk with application to Down's syndrome. Stat Med 11: 257-268.

Shi Z, Xu W, Loechel F, Wewer UM, Murphy LJ. 2000. ADAM 12, a disintegrin metalloprotease, interacts with insulin-like growth factor-binding protein-3. J Biol Chem 275: 18574-18580.

Snijders RJM, Noble P, Sebire N, Souka A, Nicolaides KH. 1998. UK multicentre project on assessment of risk for trisomy 21 by maternal age and fetal nuchal translucency thickness at 10-14 weeks of gestation. Lancet 18: 519-521.

Snijders RJM, Sundberg K, Holzgreve W, Henry G, NIcolaides KH. 1999. Maternal age and gestation specific risk for trisomy 21. Ultrasound Obstet Gynecol 13: 167-170.

Spencer K, Souter V, Tul N, Snijders R, NIcolaides KH. 1999. A screening program for trisomy 21 at $10-14$ weeks using fetal nuchal translucency, maternal serum free $\beta$-human chorionic gonadotropin and pregnancy associated plasm protein -A. Ultrasound Obstet Gynecol 13: 231-237.

Spencer K, Crossley JA, Aitken DA, Nix ABJ, Dunstan FDJ, Williams K. 2002. Temporal changes in maternal serum biochemical markers of trisomy 21 across the first and second trimester of pregnancy. Ann Clin BIochem 39: 567-576.

Spencer K, Spencer CE, Power M, Dawson C, Nicolaides KH. 2003a. Screening for chromosomal abnormalities in the first trimester using ultrasound and maternal serum biochemistry in a one-stop clinic: a review of three years prospective experience. BJOG 110: 281-286.

Spencer K, Bindra R, Nicolaides KH. 2003b. Maternal weight correction of maternal serum PAPP-A and free $\beta$-hCG when screening for trisomy 21 in the first trimester of pregnancy. Prenat Diagn 23: $851-855$.

Spencer K, Bindra R, Nix ABJ, Heath V, Nicolaides KH. 2003c. Delta-NT or NT MoM: which is the most appropriate method for calculating accurate patient specific risks for trisomy 21 in the first trimester? Ultrasound Obstet Gynecol 22: 142-148.

Spencer K, Crossley JA, Aitken DA, Nix ABJ, Dunstan FDJ, Williams K. 2003d. The effect of temporal variation in biochemical markers of trisomy 21 across the first and second trimester of pregnancy on the estimation of individual patient specific risks and detection rates for Down's syndrome. Ann Clin Biochem 40: 219-231.

van der Veen V, Beekhuis JR, Cornel MC, Mantingh A, de Walle HE, de Wolf BT. 1997. A demographic approach to the assessment of Down syndrome screening performance. Prenat Diagn 17: 717-724. 